\title{
Electro-mechanical analysis of a multilayer piezoelectric cantilever energy harvester upon harmonic vibrations
}

\author{
Zdeněk Machů${ }^{1}$, Zdeněk Majer ${ }^{1, *}$, Oldřich Ševeček ${ }^{1}$, Kateřina Štegnerová ${ }^{1}$, and Zdeněk Hadaš ${ }^{1}$ \\ ${ }^{1}$ Faculty of Mechanical Engineering, Brno University of Technology, Technická 2896/2, 61669 Brno, Czech Republic
}

\begin{abstract}
This paper addresses an important issue of the individual layer thickness influence in a multilayer piezo composite on electro-mechanical energy conversion. The use of energy harvesting systems seems to be very promising for applications such as ultra-low power electronics, sensors and wireless communication. The energy converters are often disabled due to a failure of the piezo layer caused by an excessive deformation/stresses occurring upon the operation. It is thus desirable to increase both reliability and efficiency of the electromechanical conversion as compared to standard concepts. The proposed model of the piezoelectric vibration energy harvester is based on a multilayer beam design with active piezo and protective ceramic layers. This paper presents results of a comparative study of an analytical and numerical approach used for the electro-mechanical simulations of the multilayer energy harvesting systems. Development of the functional analytical model is crucial for the further optimization of new (smart material based) energy harvesting systems, since it provides much faster response than the numerical model.
\end{abstract}

\section{Introduction}

The generation of electricity from alternative sources of energy has become a hot topic during the last years, especially in connection to Industry 4.0 applications [1-2]. A huge amount of electric energy is obtained by conversion of solar, water or wind power. In general, the energy obtained in such way is called "green energy". Another way to get "free energy" is to convert mechanical energy into electrical, especially from vibrations. This is generally referred to as energy harvesting system and is schematically illustrated in Fig. 1.

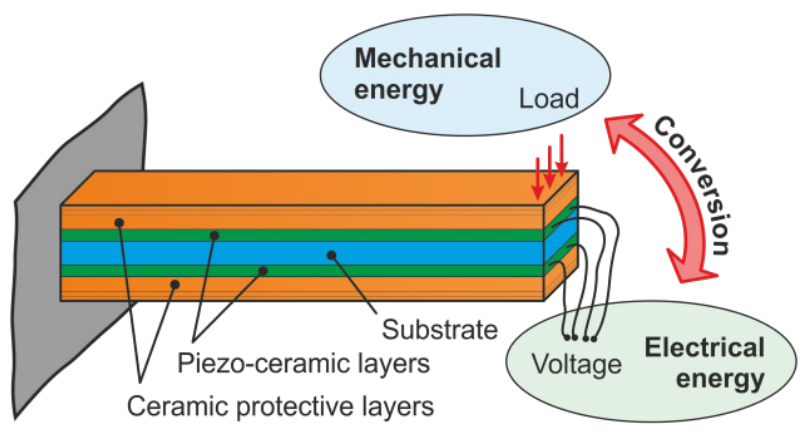

Fig. 1. The proposed new multilayer concept of energy converter with protective layers bringing pre-straining to the piezoceramics.

In this design, the standard structure of the piezoceramic energy converters is supplemented with protective layers. These layers will provide higher reliability of the piezoceramics and they will also change the electromechanical properties of piezoceramic structure, because they will introduce residual stresses (tensile or compressive) in the whole multilayer system. The outer (protective) layers, prepared from a mechanically durable material and enhanced with internal compressive stresses, can protect the inherently brittle piezoceramics and the presence of internal stresses in the piezoceramic layer can further enhance its electromechanical characteristics.

\subsection{Present state of the art}

\subsubsection{Piezoceramic material}

Lead zirconate titanate (PZT) has been dominating on the market with piezoelectric ceramics. Since these ceramics contain toxic lead, they are in the last decade often replaced by alternative materials. Nevertheless, these are usually more expensive and not easily available as PZT ceramics. The mostly used ferroelectrics replacements are e.g. the Barium Titanate (BTO), Sodium Potassium Niobate (KNN) or Bismuth Sodium Titanate (BNT) [3-7].

Generally, the piezoelectric materials nowadays are under systematic development. Piezoceramic materials suffer by inherent brittleness, which is usually one order lower as compared to the structural ceramics. Also, changes of mechanical properties during poling and/or after the application of electric field were reported [8-10].

\footnotetext{
* Corresponding author: majer@,vutbr.cz
} 


\subsubsection{Laminate composites}

Anisotropy of mechanical properties after the polarization makes the prediction of overall fracture behavior more difficult. The fracture toughness as a governing property can in one direction increase and in the other one decrease compared to a non-poled state [11-12]. The brittle fracture of glasses and ceramics is a consequence of material defects (located either within the bulk material or at the surface), induced by the processing and/or machining procedures [13-15].

Layered ceramic materials (also referred to as "ceramic laminates") are becoming very attractive in many areas of the ceramic technology due to their better and adjustable fracture mechanics characteristics in comparison with bulk ceramics. Especially by introduction of the residual stresses inside the multilayer ceramic structure, the fracture toughness, strength and mechanical reliability can be significantly changed/improved. The laminates designed with strong interfaces and compressive residual stresses have led to an increase in fracture energy, thermal shock resistance and, in some cases, a decrease in the sensitivity to the size of defects. Utilization of tailored compressive residual stresses, acting as physical barriers to crack propagation, has succeeded in many ceramic systems [16-19].

The aim of this study is to present a functional analytical model of the cantilever energy harvester with a tip mas, which will provide fast information about its natural frequency and generated electric power upon steady state or transient vibrations in the vicinity of the natural frequency. The analytical model will be compared with reference FE solution to prove its functionality. The developed model will be used in the following research for an optimization of the multilayer energy harvester from both the electrical and the fracture-mechanics point of view where influence of various levels of residual stresses in particular layers on the generated power and resistance to surface crack propagation will be taken into account.

\section{Piezoelectric energy harvester}

\subsection{Structure of the proposed model}

The considered structure of a composite laminate beam can be seen in Fig. 2. The proposed model contains seven layers in total. There are two piezoelectric layers on each side of the base material. Outside these layers,

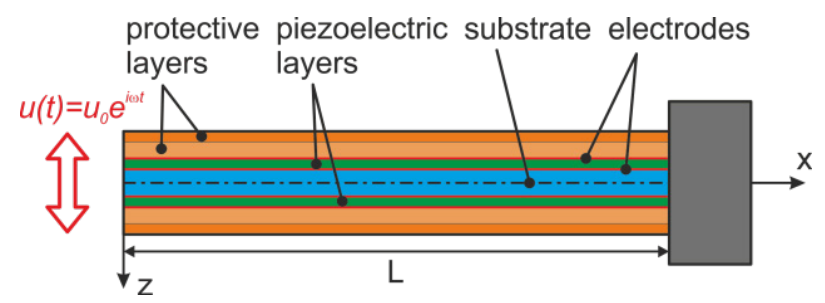

Fig. 2. Considered model of the multilayer vibrational energy harvester with a tip mass containing 2 piezo layers, 5 $\mathrm{Al} 2 \mathrm{O} 3 / \mathrm{ZrO} 2$ layers and 4 electrodes two protective layers follow. On both sides of the piezoelectric layers, the conductive electrodes are present and are used both for poling of the piezoceramics and for the subsequent collection of the electric charge.

The proposed composite laminate beam with dimensions $60 \times 10 \times 1.5 \mathrm{~mm}$ is clamped on one side and is kinematically excited by a displacement amplitude of 12 $\mu \mathrm{m}$ (by harmonic function). Moreover, a second variant with extra mass $(10 \mathrm{~g})$ at the free end was considered, see Fig. 3.

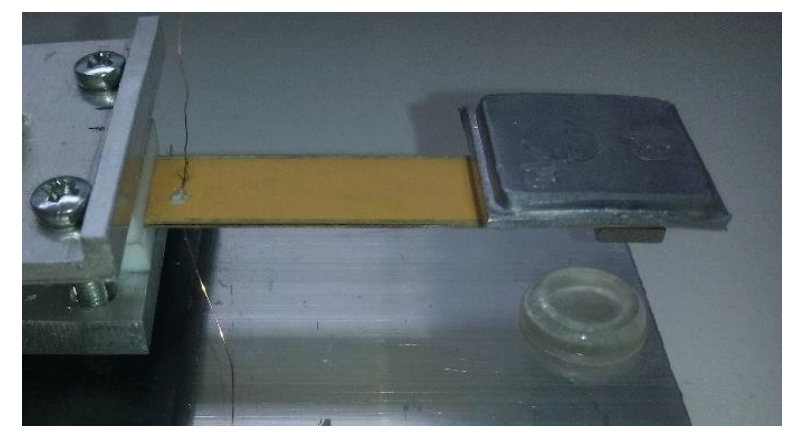

Fig. 3. Example of the real energy harvester with a tip mass.

\subsection{Analytical model}

In order to speed up parametric studies upon the searching for an optimal design of the multilayer energy harvester, its analytical model was developed and is briefly described in this section. Following assumptions were made to derive the governing equations of the analytical model:

- Euler-Bernoulli beam theory is used

- Used layer combination is symmetric

- No axial loads are present

- Linear energy conversion is assumed (the frequency of generated electrical current is proportional to the kinematic excitation forcing frequency $\omega$ )

- Electric field intensity can change linearly with zcoordinate in each layer and electric field intensity together with electric flux density has only its zcomponent, other components are being zero

- Each piezoelectric layer has its own connected resistor of value $R$

- All piezoelectric layers have the same poling direction

We assume, that the beam is subjected to pure bending, therefore mechanical strain $S$ can be expressed as:

$$
S=-\frac{\partial^{2} w}{\partial x^{2}} z
$$

The piezoelectric constitutive equations for uniaxial loading have the following form:

$$
\begin{aligned}
& S=\frac{1}{Y_{i}} T+d_{i} E, \\
& D=d_{i} T+\varepsilon_{i}^{T} E,
\end{aligned}
$$


where $Y_{i}$ is elastic modulus of $\mathrm{i}$-th layer, $T$ is the mechanical stress, $d_{i}$ is a piezoelectric charge coefficient of $\mathrm{i}$-th layer, $E$ is the electric field intensity, $D$ is the electric flux density and $\varepsilon_{i}^{T}$ is a dielectric permittivity at constant mechanical stress of i-th layer. From eq. (2) we receive the following constitutive relation:

$$
T=\left(S-d_{i} E\right) Y_{i} .
$$

Substituting eq. (4) into eq. (3) leads to:

$$
D=e_{i} S+\varepsilon_{i}^{S} E,
$$

where $e_{i}=Y_{i} d_{i}$ is piezoelectric modulus and $\varepsilon_{i}^{S}=\varepsilon_{i}^{T}-d_{i} e_{i}$ is dielectric permittivity at constant strain of the i-th layer.

For a static-structural problem, where $D=$ const.$=0$, we receive a following expression for the electric field intensity:

$$
E=-\frac{e_{i}}{\varepsilon_{i}^{S}} S=\frac{e_{i}}{\varepsilon_{i}{ }^{S}} \frac{\partial^{2} w}{\partial x^{2}} z
$$

Now we have to find expression for the electrical current $I$, generated by vibrations in each layer. It is known that:

$$
\begin{gathered}
I(t)=\frac{d Q(t)}{d t} \\
\frac{U_{i} e^{i \omega t}}{R}=\frac{d}{d t}\left(\iint_{S} D e^{i \omega t} d S\right) .
\end{gathered}
$$

Substituting eq. (5) into eq. (7) yields to:

$$
\frac{U_{i}}{R} e^{i \omega t}=i \omega\left[\int_{-\frac{B}{2}}^{\frac{B}{2}}\left(\int_{0}^{L}-e_{i} \frac{\partial^{2} w}{\partial x^{2}} z+\varepsilon_{i}^{S} E d x\right) d y\right] e^{i \omega t},
$$

where $B$ is width of the beam. For the electric flux density, it is assumed that $\partial D / \partial z=0$ (no volumetric charge density is present) and the electric field intensity is assumed to change linearly with $\mathrm{z}$-coordinate. Therefore, the voltage drop can be expressed as $U_{i}=-E\left(z_{T i}\right) h_{i}$, and we can rewrite eq. (8) as follows:

$$
\frac{U_{i}}{R} e^{i \omega t}=i \omega\left[\int_{-\frac{B}{2}}^{\frac{B}{2}}\left(\int_{0}^{L}-e_{i} \frac{\partial^{2} w}{\partial x^{2}} z_{T i}-\varepsilon_{i}^{S} \frac{U_{i}}{h_{i}} d x\right) d y\right] e^{i \omega t} .
$$

Since the beam is clamped at $x=0$, the term $\partial w / \partial x(0)$ vanishes. Thus, the expression for voltage drop in i-th layer is:

$$
U_{i}=-\frac{e_{i} \frac{\partial w}{\partial x}(L) z_{T i}}{\frac{1}{i \omega R B}+\frac{\varepsilon_{i}^{S} L}{h_{i}}} .
$$

Now we have to find an expression for the electric field intensity, which changes linearly with z-coordinate and satisfies condition $U_{i}=-E\left(z_{T i}\right) h_{i}$. Eq. (11) meets both criteria:

$$
\begin{aligned}
E(z)= & \frac{e_{i}}{h_{i}\left(\frac{1}{i \omega R B}+\frac{\varepsilon_{i}^{S} L}{h_{i}}\right)} \frac{\partial w}{\partial x}(L) z_{T i}+ \\
& +\frac{e_{i}}{\varepsilon_{i}^{S}} \frac{\partial^{2} w}{\partial x^{2}}\left(z-z_{T i}\right)
\end{aligned}
$$

Now we can form the new constitutive law by substituting eqs. (1) and (11) into eq. (4):

$$
T=\left(-\frac{\partial^{2} w}{\partial x^{2}} z-\zeta_{i} \frac{\partial w}{\partial x}(L) z_{T i}-\Psi_{i} \frac{\partial^{2} w}{\partial x^{2}}\left(z-z_{T i}\right)\right) Y_{i},
$$

where

$\zeta_{i}=\frac{d_{i} e_{i}}{h_{i}\left(\frac{1}{i \omega R B}+\frac{\varepsilon_{i}^{S} L}{h_{i}}\right)}$ and $\Psi_{i}=\frac{d_{i} e_{i}}{\varepsilon_{i}^{S}}$

represent the piezoelectric feedback of each layer.

In order to derive the differential equation of motion, an expression for the potential energy must be formed:

$$
E_{p}=\frac{1}{2} \iiint_{V} T S d V=\frac{1}{2} \int_{0}^{L}\left\{J_{y}^{*}\left(\frac{\partial^{2} w}{\partial x^{2}}\right)^{2}\right\} d x+\frac{1}{2} \alpha\left(\frac{\partial w}{\partial x}(L)\right)^{2},
$$

where

$$
J_{y}{ }^{*}=B \sum_{i=1}^{N}\left(\frac{1}{3} Y_{i}\left(z_{i+1}{ }^{3}-z_{i}^{3}\right)+\frac{1}{3} Y_{i} \Psi_{i}\left(z_{i+1}{ }^{3}-z_{i}^{3}\right)-Y_{i} \Psi_{i} z_{T i}{ }^{2} h_{i}\right)
$$

is the bending stiffness of a layered beam with piezo stiffening effect and $\alpha=B \sum_{i=1}^{N}\left(Y_{i} \zeta_{i} z_{T i}{ }^{2} h_{i}\right)$ is the piezoelectric bending moment.

The expression for kinetic energy, which also accounts for the added discrete mass $M$ at the beam tip, is given as follows:

$$
\begin{aligned}
& E_{k}=\frac{1}{2} \iiint_{V} \rho_{i}\left(\frac{\partial w}{\partial t}\right)^{2} d V+\frac{1}{2} M\left(\frac{\partial w}{\partial t}(L)\right)^{2}= \\
& =\frac{1}{2} \int_{0}^{L} m^{*}\left(\frac{\partial w}{\partial t}\right)^{2} d x+\frac{1}{2} M\left(\frac{\partial w}{\partial t}(L)\right)^{2}
\end{aligned}
$$

Here $m^{*}=B \sum_{i=1}^{N} \rho_{i} h_{i}$ is the mass of a layered beam per unit of length. Using Hamilton's principle $\left(\int_{t_{1}}^{t_{2}} \delta\left(E_{k}-E_{p}\right) d t=0\right)$ we obtain a differential equation of motion (15) with boundary conditions (16):

$$
J_{y}^{*} \frac{\partial^{4} w}{\partial x^{4}}+m^{*} \frac{\partial^{2} w}{\partial t^{2}}=0
$$




$$
\begin{aligned}
& w(x=0)=u_{0} \\
& \frac{\partial w}{\partial x}(x=0)=0 \\
& \frac{\partial^{2} w}{\partial x^{2}}(x=L)=-\frac{\alpha}{J_{y}^{*}} \frac{\partial w}{\partial x}(x=L) \\
& \frac{\partial^{3} w}{\partial x^{3}}(x=L)=\frac{M}{J y^{*}} \frac{\partial^{2} w}{\partial t^{2}}(x=L)
\end{aligned}
$$

In order to account for damping, the eq. (15) must be extended with a term representing the stiffness part of Rayleigh's damping:

$$
J_{y}^{*} \frac{\partial^{4} w}{\partial x^{4}}+\frac{2 b_{r}}{\Omega_{0}} J_{y}^{*} \frac{\partial^{5} w}{\partial x^{4} \partial t}+m^{*} \frac{\partial^{2} w}{\partial t^{2}}=0,
$$

where $\Omega_{0}$ represents the first undamped natural frequency and $b_{r}$ is a damping ratio. The general solution of eq. (17) is:

$$
w(x)=C_{1} \cosh (\beta x)+C_{2} \sinh (\beta x)+C_{3} \cos (\beta x)+C_{4} \sin (\beta x),
$$

where $\beta=f(\omega)=\sqrt[4]{\frac{m^{*} \omega^{2}}{J_{y}^{*}\left(1+i \frac{2 b r}{\Omega_{0}} \omega\right)}}$.

To find natural frequencies of the given beam configuration, one has to solve the following system of linear equations:

$$
\begin{aligned}
& w(x=0)=0 \\
& \frac{\partial w}{\partial x}(x=0)=0 \\
& \frac{\partial^{2} w}{\partial x^{2}}(x=L)=-\frac{\alpha}{J_{y}^{*}} \frac{\partial w}{\partial x}(x=L) \\
& \frac{\partial^{3} w}{\partial x^{3}}(x=L)=\frac{M}{J y^{*}} \frac{\partial^{2} w}{\partial t^{2}}(x=L)
\end{aligned}
$$

Since no external load is present in the system (19), the non-trivial solution can only be obtained by rendering the system underdetermined (i.e. its system matrix is singular). The resulting transcendental eq. (20) can be used to extract natural frequencies of the given beam configuration:

$$
\begin{gathered}
\beta[1+\cosh (\beta L) \cos (\beta L)]+ \\
+\beta^{2} \frac{M}{m^{*}}[\sinh (\beta L) \cos (\beta L)-\cosh (\beta L) \sin (\beta L)]+ \\
+\frac{\alpha}{J_{y}^{*}}[\cosh (\beta L) \sin (\beta L)+\sinh (\beta L) \cos (\beta L)]+ \\
+\beta \frac{M \alpha}{m^{*} J_{y}^{*}}[\cosh (\beta L) \cos (\beta L)-1]=0
\end{gathered}
$$

where $\beta$ is a function being dependent on the complexvalued natural frequency $\Omega^{*}$ :

$$
\beta=f\left(\Omega^{*}\right)=\sqrt[4]{\frac{m^{*}\left(\Omega^{*}\right)^{2}}{J_{y}^{*}\left(1+i \frac{2 b r}{\Omega_{0}} \Omega^{*}\right)}} .
$$

\subsection{FE numerical model}

To verify outputs of the analytical model, a numerical (FE) analysis of the laminate composite was simultaneously performed (using the commercial FE system ANSYS). The created FE model contains more than 1000 three-dimensional quadratic brick elements of three types. SOLID186 is used for all composite layers except piezo layers, which are meshed using the SOLID226 elements. CIRCU94 elements are used to simulate resistor connected to piezo layers. The boundary conditions (BCs) in the case of modal analysis were prescribed at the fixed end of the beam (as zero displacements in all directions) and in the case of harmonic analysis, the displacement $\mathrm{BC}$ at the fixed end in the Z-direction was prescribed as an amplitude of time-harmonic displacement (see also Fig 2 in section 2.1).

\section{Results}

One of the main objective of this work was to perform a comparative study of the analytical and numerical model of the vibrational energy harvester, especially in terms of the calculated natural frequency, transverse displacement of the proposed composite laminated beam and the acquired voltage $U$, presented here in the form of electric potential $\phi$ at the interface between each layer $U=\Delta \phi$. The representative results for two selected configurations are presented (resistance of the electrical load $R=1 \mathrm{k} \Omega$ and $1 \mathrm{M} \Omega$ ).

It is well known that the maximum output voltage (power) is achieved at resonance frequencies. In the present study, only the first natural frequency was considered and used to analyze the obtained voltage output, since upon this frequency the obtained voltage should be maximal. Both analytical and numerical results of the natural frequency calculations are shown in the Table 1 . Here it is obvious that by adding extra mass to the end of the suggested beam, the "working range" (the resonance frequency) of the designed beam can be significantly influenced (in our case almost three times). One should also point out an excellent match in determination of the first natural frequency between both approaches.

Table 1. Results from the modal analysis, $1^{\text {st }}$ natural frequency.

\begin{tabular}{|c|c|c|c|c|}
\hline & \multicolumn{2}{|c|}{$R=1 k \Omega$} & \multicolumn{2}{c|}{$R=1 M \Omega$} \\
\hline & $\mathrm{M}=0 \mathrm{~g}$ & $\mathrm{M}=10 \mathrm{~g}$ & $\mathrm{M}=0 \mathrm{~g}$ & $\mathrm{M}=10 \mathrm{~g}$ \\
\hline $\begin{array}{c}\text { Matlab } \\
\text { (Analytical } \\
\text { model) }\end{array}$ & $414.9 \mathrm{~Hz}$ & $140.8 \mathrm{~Hz}$ & $416.1 \mathrm{~Hz}$ & $141.3 \mathrm{~Hz}$ \\
\hline $\begin{array}{c}\text { Ansys } \\
\text { (Numerical } \\
\text { model) }\end{array}$ & $419.5 \mathrm{~Hz}$ & $142.2 \mathrm{~Hz}$ & $419.5 \mathrm{~Hz}$ & $142.2 \mathrm{~Hz}$ \\
\hline
\end{tabular}

The transverse displacement of beam's centerline along beam's length for different tip mass is depicted in the Fig. 4. One can see that the displacement in this case 
is practically independent of the electric load resistance $R$, since the piezoelectric stiffening effect on the whole composite structure is somewhat negligible. The smaller deflections in the case of added mass are due to lower value of clamped end's acceleration amplitude $\left(1^{\text {st }}\right.$ natural frequency), which is governed by relation $a=u_{0} \omega^{2}$ (note that the value of $u_{0}$ remains the same for all studied configurations).

The voltage output for each configuration is shown in the Fig. 5. It can be seen that the voltage output tends to increase with resistive load, which agrees with basic principles of the electrical theory. Due to same poling direction of both piezoelectric layers and the same distance from the beam centerline, the voltage generated in both piezoelectric layers has the same value but an opposite sign. The lower values of generated voltage in the case of beam with an added mass are caused by a smaller deflection of the beam's free end (see eq. (10)) The smaller deflection is caused by the lower natural frequency and thus lower applied acceleration in the zdirection on the beam's fixed end during the simulation.
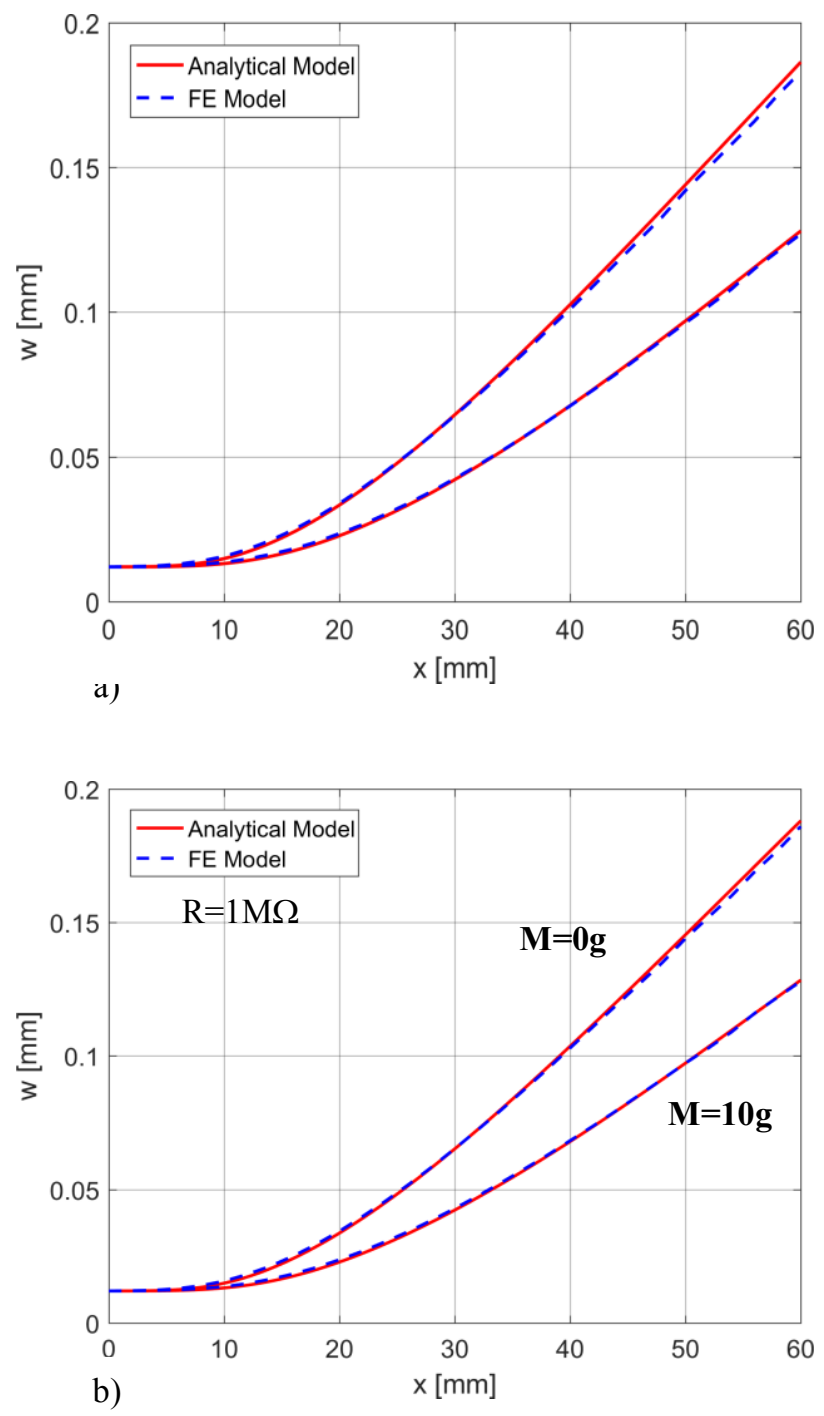

Fig. 4. Displacement calculated along the beam's centerline for various tip mass cases and resistance of the electrical load: a) $\mathrm{R}=1 \mathrm{k} \Omega$, b) $\mathrm{R}=1 \mathrm{M} \Omega$.

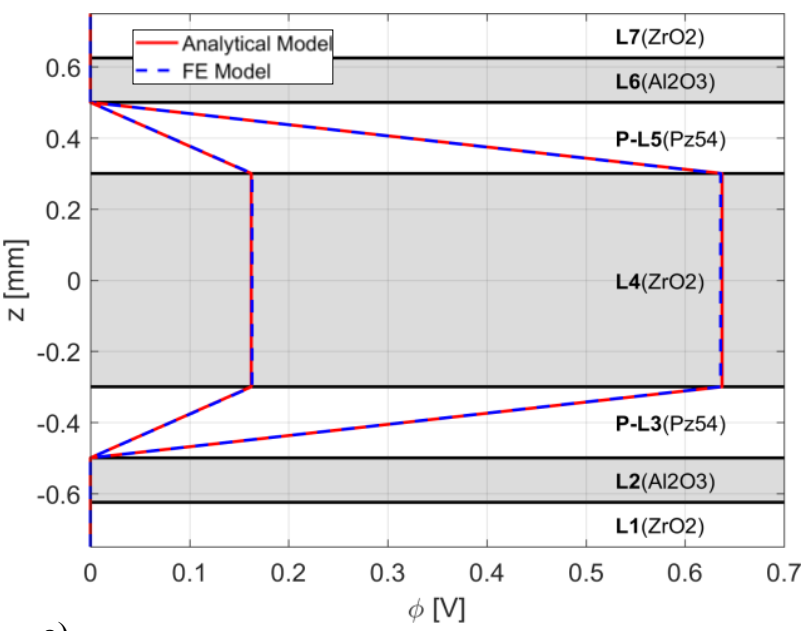

a)

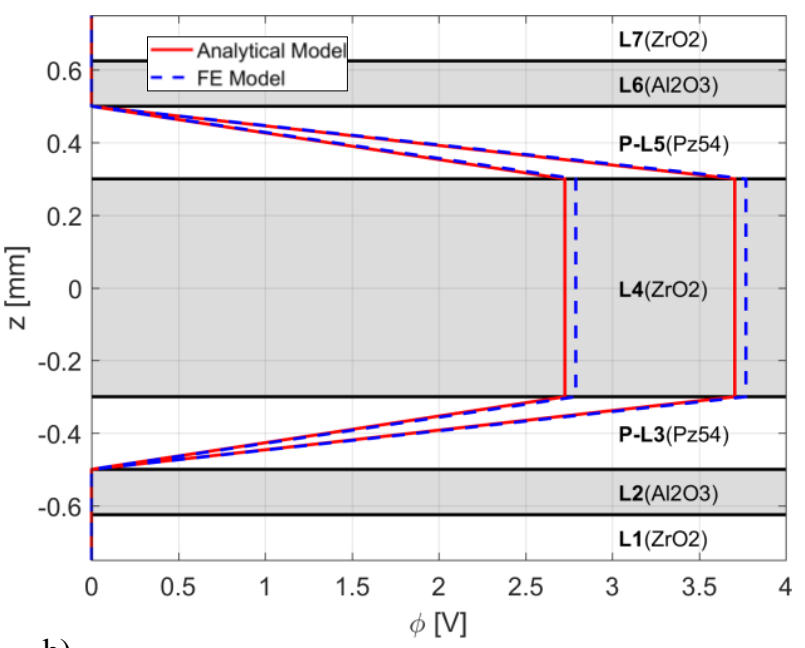

b)

Fig. 5. Electric potential values of the designed laminate for various tip mass and resistance of the electrical load: a) $R=1 \mathrm{k}$ $\Omega, \mathrm{b}) \mathrm{R}=1 \mathrm{M} \Omega$.

\section{Summary}

In this work, a comparison of analytical and FE solution of vibrating composite laminate beam containing active piezoelectric layers was performed. Natural frequencies and steady-state response during resonance were analyzed by both these models with a very good agreement in the obtained results. Small discrepancies in the case of steady-state results predicted by both models are caused by the mismatch in natural frequencies between analytical and FE model.

The currently performed analysis proved a very good functionality of the developed analytical model of the vibrating multilayer piezoelectric energy harvester and the developed analytical model will be used in the following research for the optimization of the distribution of particular layers within the laminate height in order to receive maximal resistance of outer protective layers to fracture and simultaneously to obtain highest possible electrical performance of the newly designed harvester. To this end, the presence of residual 
thermal stresses in individual layers (induced by the mismatch in coefficients of thermal expansion of the used materials) will be also utilized.

The proposed multilayer energy harvesters can be e.g. used to be placed on the large vibrating structures such as aircraft wings, bridges or windmill to generate electrical power especially for self-diagnostic purposes of the considered structure. Finally, it is a very promising area of application to railway, especially in the rail track.

This research was supported through a grant number 17-08153S of the Czech Science Foundation.

\section{References}

1. O.B. Digilina, I.B. Teslenko, N.V. Abdullaev, Adv. in Int. Systems and Computing 726, 32 (2019)

2. O. Andrs, Adv. in Int. Systems and Computing 644, 149 (2018)

3. Y. Saito, H. Takao, T. Tani, T. Nonoyama, K. Takatori, T. Homma, T. Nagaya, M. Nakamura, Nature 432, 84 (2004)

4. R.Z. Zuo, X.S. Fang, C. Ye, Appl. Phys. Letters 90, 092904 (2007)

5. I. Logothetis, D. Matsouka, S. Vassiliadis, C. Vossou, E. Siores, J. of Elect. Mat. 47, 3709 (2018)

6. M.T. Todaro, F. Guido, V. Mastronardi, D. Desmaele, G. Epifani, L. Algieri, M. De Vittorio, Microelectronic Eng. 183-184, 23 (2017)

7. S.B. Lang, Ferroelectrics 332, 227 (2016)

8. S. Rattanachan, Y. Miyashita, Y. Mutoh, J. Eur. Cer. Soc. 24, 775 (2004)

9. X. Liu, S. Xue, J. Ma, J. Zhai, B. Shen, F. Wang, X. Zhao, H. Yan, J. Eur. Cer. Soc. 38, 4631 (2018)

10. K. Prabahar, R. Ranjith, A. Srinivas, S.V. Kamat, B. Mallesham, V.L. Niranjani, J.P. Praveen, D. Das, Ceramics Int. 43, 5356 (2017)

11. R. Bermejo, H. Grünbichler, J. Kreith, C. Auer, J. Eur. Cer. Soc. 30, 705 (2010)

12. V. Carollo, J. Reinoso, M. Paggi, J. Eur. Cer. Soc. 38, 2994 (2018)

13. R. Morrell, Fractography of Brittle Materials (National Physical Laboratory, Teddigton, 1999)

14. D. Leguillon, E. Martin, O. Sevecek, R. Bermejo, Int. J. of Fracture 212, 89 (2018)

15. D.M. Chang, B.L. Wang, Phil. M. 94, 2633 (2014)

16. V.M. Sglavo, M. Bertoldi, Acta Mat. 54, 4929 (2006)

17. K. Maca, V. Pouchly, D. Drdlik, H. Hadraba, Z. Chlup, J. Eur. Cer. Soc. 37, 4287 (2017)

18. Y. Chang, R. Bermejo, O. Ševeček, G.L. Messing, J. Eur. Cer. Soc. 35, 631 (2015)

19. K. Štegnerová, L. Náhlík, P. Hutař, Key Eng. Mat. 665, 93 (2016) 